Received: 25.12 .2017

Revised: 26.03.2018

Accepted: 30.03 .2018

DOI: $10.17804 / 2410-9908.2018 .2 .006-028$

\title{
MECHANICS OF SHELLS AND ITS APPLICATIONS
}

\author{
I. G. Emelyanov \\ Institute of Engineering Science, Ural Branch of the Russian Academy of Sciences, \\ 34 Komsomolskaya St., 620049, Ekaterinburg, Russia \\ $\underline{\text { https://orcid.org/0000-0002-9733-5485; ه emelyanov@imach.uran.ru }}$ \\ Corresponding author. E-mail: emelyanov@imach.uran.ru \\ Address for correspondence: ul. Komsomolskaya 34, 620049, Ekaterinburg, Russian Federation \\ Tel.: +7 (343) 37535 73; fax: +7 (343)374 5330
}

The paper presents the results of applying shell theory methods to solving problems of determining the stress-strain state and durability of thin-walled structures. Some basic concepts and principles of the shell theory are given, as well as some information on its history. A relationship is shown between the construction of a mathematical model of a certain class of problems for shells and the development of a problem solution method. Frequently used shell models and numerical methods for solving applied problems are considered. A way of reducing problem dimensionality is considered; namely, the application of the method of reducing the equations of the theory of shells to a number of Cauchy problems with Godunov orthogonalization. It is shown that one type of problems for shells, which is often encountered in the analysis of various technical objects, is problems with moving boundaries, particularly, contact problems. The paper considers one of the effective ways of solving contact problems for shells of revolution, namely, the virtual element method. In contrast to the finite element method, an increase in the number of virtual elements does not increase the size of the structure stiffness matrix. This is a great advantage of the virtual element method, which allows one to save computational resources for many problems. The study presents scientific papers on the investigation of thin-walled structures and results obtained with allowance made for the use of application of recent methods of shell mechanics.

Keywords: shell elements, shell theory, evaluation of strength and durability, contact problems, problem dimensionality reduction, numerical methods, and virtual element method.

\section{References}

1. Lyav A. Matematicheskaya teoriya uprugosti [Mathematical Theory of Elasticity]. Moscow, Leningrad, ONTI Publ., 1935, 674 p. (In Russian).

2. Vlasov V.Z. Obshchaya teoriya obolochek $i$ ee prilozheniya $v$ tekhnike [General Theory of Shells and its Application in Engineering]. Moscow, Leningrad, Gostekhizdat Publ., 1949, 784 p. (In Russian).

3. Goldenveyzer A.L. Teoriya uprugikh tonkikh obolochek [Theory of Elastic Thin Shells]. M., Nauka Publ., 1976, 512 p. (In Russian).

4. Lur'e A. I. Statics of thin-walled elastic shells, transl. from Russian, ed. [AEC-tr-3798, U.S. Atomic Energy Commission]. Tech. Info. Service, 1947.

5. Novozhilov V.V. Teoriya tonkikh obolochek [Theory of Thin-Walled Shells]. Leningrad, Sudostroenie Publ., 1962, 431p. (In Russian).

6. Timoshenko S.P., Woinowsky-Krieger S. Theory of Plates and Shells. McGraw-Hill Book Company Inc., 1959. 
7. $\quad$ Flügge W. Statik und Dynamik der Schalen, 2-te neubearb. Aufl. Springer-Verl., 1957.

8. Chernykh K.F. Lineynaya teoriya obolochek [Linear Theory of Shells. Part I]. Leningrad, LGU Publ., 1962, 274 p. (In Russian).

9. Chernykh K.F. Lineynaya teoriya obolochek [Linear Theory of Shells. Part II]. Leningrad, LGU Publ., 1964, 396 p. (In Russian).

10. Grigorenko Ya.M., Vasilenko A.T. Metody rascheta obolochek. T. 4. Teoriya obolochek peremennoy zhestkosti [Shell Calculation Methods. Vol. 4. Theory of Variable-Stiffness Shells]. Kiev, Nauk. Dumka, 1981, 544 p. (In Russian).

11. Volmir A.S., Kuranov B.A., Turbaivsky A.T. Statika i dinamika slozhnykh struktur [Statics and Dynamics of Complex Structures]. Moscow, Mashinostroenie Publ., 1989, 248 p. (In Russian).

12. Grigorenko Ya.M. Izotropnye $i$ anizotropnye sloistye obolochki vrashcheniya peremennoy zhestkosti [Isotropic and anisotropic layered shells of revolution with a variable stiffness]. Kiev, Naukova Dumka Publ., 1973, 228 p. (In Russian).

13. Rogalevich V.V. Kollokatsionnye metody. Sushchnost. Primery [Collocation Methods. Essence. Examples]. Ekaterinburg, AMB Publ., 2001, 298 p. (In Russian).

14. Crouch S. L., Starfield A. M. Boundary element methods in solid mechanics. George Allen \& Unwin, London, 1983, 322 p.

15. Brebbia C.A., Walker S. Boundary Element Techniques in Engineering. NewnesButterworths, London, 1979.

16. Artyukhin Yu.P., Gribov A.P. Resheniye zadach nelineynogo deformirovaniya plastin i pologikh obolochek metodom granichnykh elementov [Solution of Nonlinear Deformation Problems by the Boundary Element Method]. Kazan, Fen Publ., 2002, 199 p. (In Russian).

17. Banerjee P. K., Butterfield R. Boundary Element Methods in Engineering Science. McGrawHill Book Company, London, 1981.

18. Kantorovich L.V., Krylov V.I. Approximate Methods of Higher Analysis. Mineola, New York, Dover Publications, Inc., 2018, 704 p.

19. Grigorenko Ya.M., Vasilenko A.T., Emelyanov I.G., Kryukov N.N., Nemish Yu.N., Pankratova N.D., Pelekh B.L., Vlaikov G.G., Maksimuk A.V., Urusova G.P., A.N. Guz ed. Mekhanika kompozitov: v 12 t. T. 8. Statika elementov konstruktsiy [Composite Mechanics. In 12 vols. Vol. 8. Statics of Structural Components]. Kiev, Naukova Dumka Publ., 1999, 379 p. (In Russian).

20. Karmishin A.V., Lyaskovets V.A., Myachenkov V.I., Frolov A.N. Statika i dinamika tonkostennykh obolochechnykh konstruktsiy [Statics and dynamics of thin-walled shell structures]. Moscow, Mashinostroenie Publ., 1975, 376 p. (In Russian).

21. Godunov S.K. Numerical solution of boundary-value problems for a system of linear ordinary differential equations. Uspekhi Matematicheskikh Nauk, 1961, vol. 16, no. 3, pp. 171-174.

22. Grigorenko Ya.M., Vlaikov G.G., Grigorenko A.Ya. Chislenno-analiticheskoye resheniye zadach mekhaniki obolochek na osnove razlichnykh modeley [Numerical Analytical Solution of Shell Mechanics Problems on the Basis of Different Models]. Kiev, Akademperiodika Publ., 2006, 472 p. (In Russian).

23. Emelyanov I.G. Kontaktnye zadachi teorii obolochek [Contact Problems of the Shell Theory]. Ekaterinburg, UrO RAN Publ., 2009, 185 p. (In Russian).

24. Emelyanov I.G., Mironov V.I. Dolgovechnost obolochechnykh konstruktsiy [Durability of Shell Structures]. Ekaterinburg, UrO RAN Publ., 2012, 224 p. (In Russian).

25. Gallagher R.H. Finite Element Analysis Fundamentals. Prentice Hall, Englewood Cliffs, New Jersey, 1975.

26. Golovanov A.I., Tyuleneva O.N., Shigabutdinov A.F. Metod konechnykh elementov v statike $i$ dinamike tonkostennykh konstruktsii [Finite Element Method in Thin-Walled Structure Statics and Dynamics]. Moscow, Fizmatlit Publ., 2006, 392 p. (In Russian).

27. Rikards R.B. Metod konechnykh elementov $v$ teorii obolochek $i$ plastin [Finite Element Analysis in Theory of Shells and Plates]. Riga, Zinatne Publ., 1988, 284 p. (In Russian). 
28. Zenkevich O.K. Metod konechnykh elementov $v$ tekhnike [Boundary-Element Method in Engineering]. Moscow, Mir Publ., 1975, 541p. (In Russian).

29. Novozhilov I.I. Voprosy mekhaniki sploshnoy sredy [Issues of Continuum Mechanics]. Leningrad, Sudostroenie Publ., 1989, 400 p. (In Russian).

30. Emelyanov I.G., Efimov V.P., Kuznetsov A.V. A model of the stress-strain state of a tank car boiler with an improved scheme of support on the frame. Tyazheloe Mashinostroyenie, 2005, no. 8, pp. 44-49. (In Russian).

31. Emelyanov I.G., Mironov V.I., Kuznetsov A.V. Evaluation of the stress state and service life of shell structures. Problemy Mashinostroeniya i Nadezhnosti Mashin, 2007, no. 5, pp. 57-65. (In Russian).

32. Vasilenko A.T., Golub G.P., Grigorenko Ya.M. Calculating the parameters of the stress state of composite structural components on the basis of shell models. In: Raschety na prochnost, vyp. 30 [Strength Calculations, iss. 30]. Moscow, Mashinostroenie Publ., 1989, pp. 87-96. (In Russian).

33. Johnson K.L. Contact Mechanics. Cambridge University Press, Cambridge, 1985.

34. Hertz H. Über die Berührung fester elastischer Körper. Journal für die reine und angewandte Mathematik, 1881, num. 92, ss. 156-171.

35. Grigolyuk E.I., Tolkachev V.M. Kontaktnyye zadachi teorii plastin i obolochek [Contact Problems in Plates and Shells Theory]. Moscow, Mashinostroeniye Publ., 1980, 411p. (In Russian).

36. Kantor B.Ya. Kontaktnye zadachi nelineynoy teorii obolochek vrashcheniya [Contact Problems in Nonlinear Theory of Rotated Shells]. Kiev, Naukova dumka Publ., 1990, 136 p. (In Russian).

37. Artyukhin Ju.P., Malkin S.A. Artjuhin Ju.P., Malkin S.A. Analiticheskie i chislennye metody resheniya integralnykh uravneniy $v$ zadachakh uprugogo vozdeistviya tel [Analytical and Numerical Methods for Solving Integral Equations in Problems of Elastic Action of Bodies]. Kazan, Kazanskiy Gos. Un-t Publ., 2007, 292 p. (In Russian).

38. Mossakovskii, V.I., Gudramovich, B.C., and Makeev, E.M. Kontaktnoe vzaimodeistvie elementov obolochechnykh konstruktsii [Contact Interaction of Shell Elements]. Kiev, Naukova dumka Publ., 288 p. (In Russian).

39. Obraztsov I.F., Nerubailo B.V., Olshansky V.P. Obolochki pri lokalizovannykh vozdeistviyakh (obzor rabot, osnovnye rezultaty i napravleniya issledovaniy). Dep. v VINITI 12.02.88 [Shells under Local Effects. Survey, Main Results and Lines of Research]. Moscow, VINITI Publ., 1988, 192 p. (In Russian).

40. Pelekh B.L., Maksimuk A.V., Korovaichuk I.M. Kontaknye zadachi dlya sloistykh elementov konstruktsiy i tel s pokrytiyami [Contact Problems for Layered Structural Components and Coated Bodies]. Kiev, Naukova Dumka Publ., 1988, 280 p. (In Russian).

41. Emelyanov I.G., Kuznetsov A.V. Application of Virtual Elements to the Determination of the Stress State of Shells of Revolution. Vychislitelnaya Mekhanika Sploshnykh Sred, 2014, vol. 7, no. 3, pp. 245-252. (In Russian).

42. Emel'yanov I.G. Investigation into the contact interaction between shell and base with notches. Journal of Machinery Manufacture and Reliability, 2015, vol. 44, no. 3, pp. 263-270. DOI: $10.3103 / \mathrm{S} 1052618815030048$.

43. Emel'yanov I.G., Mironov V.I., Kuznetsov A.V. Evaluation of the life of a shell construction lying on supports. Journal of Machinery Manufacture and Reliability, 2010, vol. 39, iss. 1, pp. 83-88. DOI: 10.3103/S1052618810010139.

44. Mironov V.I., Yemel'yanov I.G. Complete diagram method for fatigue resistance calculation. In: Trudy mezhdunarodnoy nauchno-tekhnicheskoy konferentsii "Prochnost materialov $i$ elementov konstruktsiy" [Strength of materials structure elements: Proceedings of International Scientific and Technical Conference, Kiev, September 28-30, 2010]. Kiev, IPP NANU, 2011, pp. 697-704. 
45. Emel'yanov I.G., Mironov V.I., Kuznetsov A.V. Cyclic life of tank car shell. In: Trudy mezhdunarodnoy nauchno-tekhnicheskoy konferentsii "Prochnost materialov i elementov konstruktsiy" [Strength of materials structure elements: Proceedings of International Scientific and Technical Conference, Kiev, September 28-30, 2010]. Kiev, IPP NANU, 2011, pp. 836-843.

46. Yemelyanov I.G., Mironov V.I., Kuznetsov A.V., Yakushev A.V. Contact interaction of boiler at the tank-truck with the tracks bearings. Izvestiya Samarskogo Nauchnogo Tsentra RAN, vol. 13, nos. 1-2, 2011, pp. 436-439. (In Russian).

47. Yemelyanov I.G. Mironov V.I., Yakushev A.V. Contact problem in the fatigue strength calculation of tank wagon elements. Transport Urala, 2011, no. 3 (30), pp. 49-55.

48. Yemelyanov I.G., Mironov V.I., Yakushev A.V., Lukashuk O.A. Development of rapid method for car steel quality. Transport Urala, 2012, no. 2 (33), pp .13-17. (In Russian).

49. Barashkova E., Emelyanov I. Stress State of Shells under Arbitrary Load. In: Proceedings of the 5th WSEAS International Conference on Finite Differences - Finite Elements - Finite Volumes - Boundary Elements (F-and-B'12), Prague, Czech Republic, September 24-26, 2012. Prague, WSEAS Press, 2012, pp.33-37.

50. Emelyanov I. G., Mironov V. I. Contact problem for a shell considering the transverse load. Journal of Machinery Manufacture and Reliability, 2013, vol. 42, no. 1, pp. 36-40. DOI: $10.3103 / \mathrm{S} 1052618813010056$.

51. Barashkova Ye.A., Yemel'yanov I.G. Determining stress state of shells at local loading. In: Matematicheskiye metody $i$ metody optimizatsii $v$ mashinostroyenii: materialy I Mezhdunarodnoy konferentsii po metodam optimizatsii v tekhnike (OTENG '13) [Mathematical methods and optimization methods in mechanical engineering: Proceedings of the Ist International Conference on Optimization Methods in Engineering, Antaliya, Turkey, October 8-10, 2013, pp.109-113.

52. Yemelyanov I.G., Kuznetsov A.V., Mironov V.I. Mathematical model describing the stress state of gas turbine locomotive cabin upon collision with obstacle. Transport Urala, 2013, no. 4 (39), pp. 71-74. (In Russian).

53. Emelyanov I. G., Kuznetsov A. V. The stressed state of shell structures under local loads. Journal of Machinery Manufacture and Reliability, 2014, vol. 43, no. 1, pp. 42-47. DOI: $10.3103 / \mathrm{S} 1052618814010051$.

54. Yemel'yanov I.G., Kuznetsov A.V., Mironov V.I. Determination of lifetime of tank car structural elements. Reliability and life of machines and structures, 2014, iss. 38, pp. 45-54.

55. Mironov V.I., Kuznetsov A.V., Emel'yanov I.G. Consideration of cyclic degradation of the material and abnormality of the surface layer mechanical properties in calculating the life of a plate with an opening. Strength of Materials, 2014, vol. 46, no. 5, pp. 638-643. DOI: 10.1007/ s11223-014-9594-y.

56. Yemel'yanov I.G. Application of discrete Fourier series to the stress analysis of shell structures. Computational Continuum Mechanics, 2015, vol. 8, no. 3, pp. 245-253. DOI: 10.7242/ 1999-6691/2015.8.3.20.

57. Emel'yanov I.G. Mironov V.I. Kuznetsov A.V. Estimation of the fracture strength of a spatial beam-rod structure notches. Journal of Machinery Manufacture and Reliability, 2015, vol. 44, no. 5, pp. 449-454. DOI: 10.3103/S1052618815050076.

58. Emel'yanov I. G. Mironov V.I. Kuznetsov A.V. On an approach to the evaluation of the strength of a spatial rod system under impact loading. Diagnostics, Resource and Mechanics of materials and structures, 2015, iss. 2, pp. 16-23. Available at http://dream-journal.org/issues/ 2015-2/2015-2_24.html (accessed 05.05.2015).

59. Emel'yanov I.G., Mironov V.I., Kuznetsov A.V. The definition of the resistance area boundaries for a locomotive operator cab under non-regulated loads. In: AIP Conf. Proc., 2016, 1785, 040014. DOI: 10.1063/1.4967071. 
60. Emel'yanov I.G. Mironov V.I. Kuznetsov A.V. Determination of safe operating conditions for supporting structures under abnormal loads. Journal of Machinery Manufacture and Reliability, 2017, vol. 46, no. 5, pp. 136-142. DOI: 10.3103/S1052618817020042.

61. Emel'yanov I.G., Mironov V.I., Kuznetsov A.V. Vehicle Survivability Calculation. Russian Journal of Construction Science and Technology, 2017, vol. 3, no. 1, pp. 40-44. DOI: 10.15826/rjcst.2017.1.005.

62. Yemel'yanov I.G., Kuznetsov A.V. Opredeleniye napryazhennogo sostoyaniya tonkostennykh konstruktsiy $\mathrm{s}$ ispol'zovaniyem metodov teorii obolochek. Transportnye Sistemy i Tekhnologii, 2017, no. 3, pp. 64-78.

63. Emelyanov I. G., Mironov V. I., Kuznetsov A. V. Evaluating the effect of damping structures in the design of a locomotive cab during a collision. AIP Conference Proceedings, 2017, vol. 1915, pp. 040011. DOI: $10.1063 / 1.5017359$. Available at: http://aip.scitation.org/toc/apc/1915/1?expanded=1915. 
Подана в журнал: 25.12 .2017

УДК 539.3

DOI: $10.17804 / 2410-9908.2018 .2 .006-028$

\title{
МЕХАНИКА ОБОЛОЧЕК И ЕЕ ПРИЛОЖЕНИЯ
}

\author{
И. Г. Емельянов
}

Федеральное государственное бюджетное учреждение науки Институт машиноведения Уральского отделения Российской академии наук, Екатеринбург, Российская Федерация

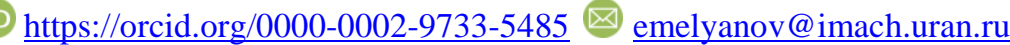

\author{
Ответственный автор. Электронная почта: emelyanov@imach.uran.ru \\ Адрес для переписки: 620049, Екатеринбург, ул. Комсомольская, 34, Российская Федерация \\ Тел.: +7 (343) 375-35-73; факс: +7 (343) 374-53-30
}

В статье представлены результаты использования методов теории оболочек для решения задач определения напряженно-деформированного состояния и долговечности тонкостенных конструкций. Приведены некоторые базовые понятия и принципы теории оболочек, а также некоторые сведения ретроспективы ее развития. Показана взаимосвязь между процессом построения математической модели какого-либо класса задач для оболочек и разработкой метода решения задачи. Рассмотрены наиболее часто используемые в настоящее время модели оболочек и численные методы решения прикладных задач, а также один из способов уменьшения размерности задачи - использование численно-аналитического метода сведения уравнений теории оболочек к ряду задач Коши с ортогонализацией по С. К. Годунову. Показано, что одной из разновидности задач для оболочек, которые часто встречаются при расчете различных технических объектов, являются задачи с подвижными границами - контактные задачи. В статье рассмотрен один из эффективных способов решения контактных задач для оболочек вращения - метод виртуальных элементов. В отличие от метода конечных элементов увеличение количества виртуальных элементов не увеличивает размер матрицы жесткости конструкции. Это большое преимущество метода виртуальных элементов, которое позволяет сохранять вычислительные ресурсы для многих задач. Приведены результаты и научные статьи по исследованию тонкостенных конструкций, полученные с учетом использования методов механики оболочек в последнее время.

Ключевые слова: тонкостенные элементы, теории оболочек, оценка прочности и долговечности, контактные задачи, уменьшения размерности задачи, численные методы, метод виртуальных элементов.

\section{1. Введение}

В настоящее время многие конструкционные элементы в инженерных конструкциях самого различного назначения, например авиационного, судостроительного, строительного, выполнены в виде тонкостенных оболочек и пластин. Экономическая эффективность таких конструкций доказана на практике. Обладая завидной легкостью, тонкостенные пространственные конструкции представляют исключительно прочную конструктивную форму. При проектировании таких тонкостенных элементов конструкций достаточно часто приходится решать задачи по оценке прочности, устойчивости, колебаниям, долговечности данных элементов конструкций. При решении прикладных задач для конструкций из тонкостенных элементов очень часто используют различные методы и подходы, которые развиваются в теории оболочек. 
Известно, что для расчета конструкционных элементов в машиностроении, один размер которых значительно меньше двух других, используются различные теории оболочек. Суть которых, заключается в том, что трёхмерные тела с помощью некоторых гипотез сводят к их двумерному представлению. С математической точки зрения это сильно упрощает решение различных прикладных задач, а вносимая погрешность для многих задач незначительна.

Поскольку в докладе будут рассматриваться тонкостенные элементы конструкций, то необходимо привести некоторые базовые понятия и принципы теории оболочек, а также некоторые сведения ретроспективы ее развития.

Первые задачи о равновесии и устойчивости пластин и оболочек ставились еще до установления общих уравнений теории упругости. В работе Огастеса Эдварда Лява [1] указывается, что эти задачи были в числе тех проблем, которые привели к созданию теории упругости.

Современная научная литература располагает огромным количеством работ, посвященных как вопросам общей теории оболочек, так и различным задачам расчета конкретных оболочечных конструкций. Основным теоретическим результатам, полученным в теории оболочек, посвящены известные монографии В. 3. Власова [2], А. Л. Гольденвейзера [3], А. И. Лурье [4], А. Лява [1], В. В. Новожилова [5], С. П. Тимошенко [6] В. Флюгге [7], К. Ф. Черных $[8,9]$.

Хотя основные уравнения и соотношения теории оболочек были получены достаточно давно, лишь для некоторых сравнительно простых классов оболочек получены аналитические решения при определенных видах нагрузки и граничных условий. Одной из характерных особенностей развития теории оболочек является взаимосвязь между процессом построения математической модели какого-либо класса задач и разработкой метода решения задач, описываемых этой моделью.

Вопросу изучения напряженно-деформированного состояния тонких оболочек при различных силовых воздействиях посвящено огромное количество публикаций. В любой из вышеперечисленных монографий по теории оболочек содержится исчерпывающее количество ссылок, которое позволяет сориентироваться по данному вопросу. Например в работе Я. М. Григоренко [10] их приведено более пятисот. Однако не всегда удается правильно оценить уникальность и научную новизну той или иной публикации ввиду большой ретроспективы данной проблематики.

С появлением вычислительной техники для решения задач определения напряженнодеформированного состояния, колебаний, устойчивости и др. различных оболочечных конструкций стали широко использоваться методы численного анализа.

Расчет сложных конструкций различного назначения при учете реальных условий эксплуатации возможен только при использовании численных методов и современной вычислительной техники.

В основном задачи теории оболочек и пластин, моделирующие тонкостенные элементы конструкций, сводятся к решению уравнений в частных производных. Эти уравнения имеют аналитические решения только для очень ограниченного круга задач для границ простой формы и простых нагрузок. Если нет возможности получить аналитическое решение задачи, то необходимо использовать численные методы.

Среди используемых в настоящее время численных методов решения инженерных задач ни один метод сам по себе не позволяет в общем случае обеспечить создание идеальных алгоритмов расчета сложной конструкции. Поэтому развитие и разработка численных методов, а также учет рационального объединения в одном алгоритме различных методов численного анализа является достаточно актуальной научной задачей. Среди наиболее часто используемых в настоящее время численных методов решения прикладных задач для тонкостенных конструкций можно выделить - метод конечных разностей $[11,12]$, метод коллока- 
ций $[11,13,14]$, метод граничных элементов $[15,16,17,18]$, методы численного интегрирования дифференциальных уравнений $[10,12,19,20]$.

При использовании методов численного интегрирования дифференциальных уравнений краевая задача должна быть сведена к одномерной. Краевая задача сводится к решению ряда задач Коши, что позволяет использовать хорошо разработанные методы интегрирования обыкновенных дифференциальных уравнений с заданными начальными условиями. Одним из таких методов является метод дискретной ортогонализации С.К. Годунова [21], который широко применяется в настоящее время для различных задач расчета тонкостенных конструкций [22, 23, 24].

Одним из самых универсальных и широко применяемых методов расчета для тонкостенных конструкций является метод конечных элементов $[25,26,27,28]$. Этот метод относится к вариационно-разностным методам и имеет в своей основе представление исходной области со сложной формой границ совокупностью простых подобластей - конечных элементов. Такое представление исследуемой области имеет смысл дискретизации континуальной задачи, т.е. замены реальной области тела с бесконечно большим числом степеней свободы приближенно-эквивалентным телом с конечным числом степеней свободы.

В настоящее время при определении напряженного состояния, устойчивости, динамики различных тонкостенных элементов широко используются вычислительные комплексы, такие как NASTRAN, COSMOS, ANSYS, LS DYNE и др., основанные на методе конечных элементов. Задача может быть решена в одномерной, двумерной или трехмерной постановке в зависимости от типа и цели задачи и располагаемой вычислительной мощности. В данных вычислительных комплексах имеются библиотеки с оболочечными конечными элементами, которые основаны на различных теориях оболочек. Если предлагается новая модель оболочки, то появляется новый конечный элемент. Этот процесс непрерывный, поскольку - как шутят «оболочечники» - оболочка неисчерпаема как атом, т. е. различные теории оболочек это какое-то новое приближение к реальному описанию континуума. По этому поводу известный советский академик В. В. Новожилов [29], писал, что при конечном числе дифференциальных уравнений теории оболочек точно описать трехмерное тело нельзя.

\section{2. Численно аналитический метод решения задач для оболочек}

Однако существует класс задач, где заложенная в вычислительных комплексах, основанных на методе конечных элементов, универсальность, теряет свою эффективность. Это происходит при решении задач для оболочек с подвижными границами, т. е. контактных задач для конструкций, взаимодействующих с жесткими или упругими телами. При решении задач методом конечных элементов для тонкостенных конструкций, взаимодействующих с другими телами, необходимо выбрать такую сетку конечных элементов, чтобы «схватить» все возможные сингулярности напряженного состояния, но при этом использовать минимально возможную размерность задачи. На рис. 1 представлена оболочка с выделенным конечным элементом.

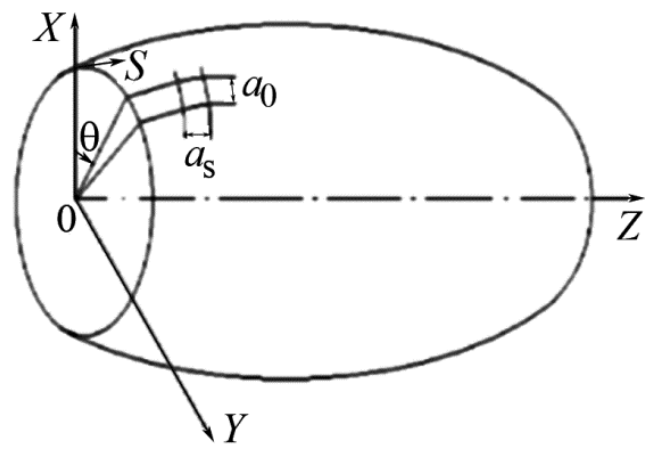

Рис. 1. Конечный элемент на оболочке вращения 
Чтобы корректно учитывать различные сингулярности, необходимо уменьшать размер конечных элементов. Уменьшение размеров элементов приводит к увеличению размеров матрицы жесткости и необходимым вычислительным мощностям, чтобы решить разрешающую систему линейных алгебраических уравнений. При решении реальных прикладных задач даже при достаточно грубой сетке количество элементов может превышать сотни тысяч. Например, при определении неосесимметричного напряженного состояния оболочки вагонацистерны, изображенной на рис. 2, потребовалось около 100 тыс. оболочечных конечных элементов типа shell [30, 31].

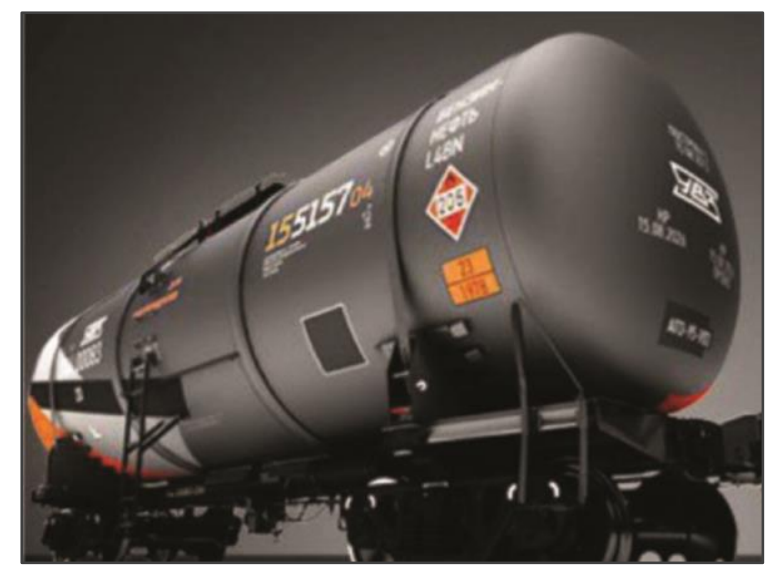

Рис. 2. Вагон цистерна

При решении такой задачи в одномерной постановке (осесимметричное напряженное состояние) необходимо около 1 тыс. элементов. При решении данной задачи в трехмерной постановке (в рамках теории упругости) необходимо не менее 2 млн объемных конечных элементов типа solid для случая, если толщина оболочки будет аппроксимирована одним конечным элементом (при необходимом соблюдении соотношений между размерами конечного элемента). Если для увеличения точности расчета толщина оболочки будет аппроксимирована тремя элементами, то число элементов возрастет до 10 млн конечных элементов. Если толщина оболочки будет аппроксимирована шестью элементами необходимо не менее 80 млн элементов. Если для первого расчетного случая для получения решения необходимо около суток, то для третьего случая речь пойдет уже о месяцах, к тому же объем вычислений будет достаточно велик и далеко не всякий компьютер сможет такое решение обработать. Поэтому вопрос уменьшения размерности задачи при решении прикладных задач для тонкостенных конструкций является весьма актуальным. Один из способов уменьшения размерности задачи - это использование численно-аналитического метода сведения уравнений теории оболочек к ряду задач Коши с ортогонализацией по Годунову.

В механике твердого деформируемого тела оболочкой принято называть тело, ограниченное двумя поверхностями, расстояние между которыми мало по сравнению с прочими его размерами. Геометрическое место точек, равноудаленных от обеих образующих оболочки поверхностей, называется срединной поверхностью оболочки. В общем случае будем рассматривать анизотропные оболочки, собранные из неоднородных слоев переменной толщины. На оболочки могут действовать неравномерные поверхностные и объемные нагрузки. Оболочки могут находиться в некотором температурном поле. Принимается, что для каждого слоя оболочки справедливы уравнения линейной теории упругости неоднородного анизотропного тела.

Для многих моделей оболочек в общем случае строится разрешающая система уравнений в частных производных. В ней в качестве искомых функций принимаются величины, входящие в произвольные граничные условия на одном из контуров координатной поверхно- 
сти оболочки. Если отнести выбранную поверхность оболочки к ортогональной системе координат $\alpha, \beta$, как на рис. 3 , где линии $\alpha=$ const, $\beta=$ const представляют собой линии главных кривизн, то такая система имеет вид $[10,32]$ :

$$
\frac{\partial \bar{N}}{\partial \alpha}=A_{0}(\alpha, \beta) \bar{N}+A_{1}(\alpha, \beta) \frac{\partial \bar{N}}{\partial \beta}+A_{2}(\alpha, \beta) \frac{\partial^{2} \bar{N}}{\partial \beta^{2}}+A_{3}(\alpha, \beta) \frac{\partial^{3} \bar{N}}{\partial \beta^{3}}+A_{4}(\alpha, \beta) \frac{\partial^{4} \bar{N}}{\partial \beta^{4}}+\bar{f}(\alpha, \beta)
$$

где $A_{0}, A_{1} \ldots . A_{4}$ - матрицы, элементы которых определяются через геометрические и жесткостные параметры оболочки; $\bar{f}$ - вектор, компоненты которого зависят от приложенных к оболочке поверхностных нагрузок и интегральных характеристик температурного поля.

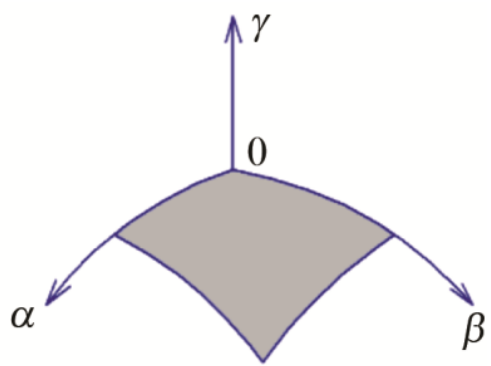

Рис. 3. Поверхность оболочки в ортогональной системе координат $\alpha, \beta$

Для классической теории оболочек, основанной на гипотезах Кирхгофа-Лява, можно использовать следующий искомый вектор восьмого порядка:

$$
\bar{N}=\left\{N_{\alpha}, \hat{S_{\alpha}}, \hat{Q}_{\alpha}, M_{\alpha}, u_{\alpha}, u_{\beta}, u_{\gamma}, \vartheta_{\alpha}\right\}
$$

где $N_{\alpha}, \hat{S_{\alpha}}, \hat{Q_{\alpha}}-$ усилия, приложенные к контуру $\alpha=$ const $M_{\alpha}$ - изгибающий момент; $u_{\alpha}$, $u_{\beta}, u_{\gamma}-$ перемещения координатной поверхности; $\vartheta_{\alpha}-$ угол поворота нормали.

Для уточненной модели, основанной на гипотезе прямой для всего пакета оболочки в целом, в уравнении $A_{3}(\alpha, \beta)=A_{4}(\alpha, \beta)=0$, а искомый вектор можно принять десятого порядка:

$$
\left\{N_{\alpha}, N_{\alpha \beta}, Q_{\alpha}, M_{\alpha}, M_{\alpha \beta}, u_{\alpha}, u_{\beta}, u_{\gamma}, \psi_{\alpha}, \psi_{\beta}\right\}
$$

где $N_{\alpha \beta}$ и $M_{\alpha \beta}$ - сдвигающее усилие, и крутящий момент, действующие на контуре $\alpha=$ const $; \psi_{\alpha}$ и $\psi_{\beta}$ - углы поворота прямолинейного элемента.

Для уточненной модели, основанной на гипотезе прямолинейного элемента, с учетом изменения его длины искомый вектор имеет вид двенадцатого порядка:

$$
\bar{N}=\left\{N_{\alpha}, N_{\alpha \beta}, Q_{\alpha}, M_{\alpha}, M_{\alpha \beta}, P_{\alpha}, u_{\alpha}, u_{\beta}, u_{\gamma}, \psi_{\alpha}, \psi_{\beta}, \psi_{\gamma}\right\},
$$

где $P_{\alpha}$ - статическая величина, которая интерпретируется как момент первого порядка от касательных напряжений поперечного сдвига по толщине пакета оболочки; $\psi_{\gamma}-$ величина, характеризующая поперечное обжатие оболочки.

Аналогично можно представить разрешающие системы уравнений и для других вариантов уточненных моделей. 
При исследовании оболочек вращения (рис. 1), в рамках классической модели, численно аналитическим методом, систему дифференциальных уравнений в частных производных можно привести к виду, который позволяет разделить переменные $s$ и $\theta[10,19,23]$ :

$$
\frac{\partial \bar{Y}}{\partial s}=\sum_{m=0}^{4} A_{m}(s, \theta) \frac{\partial^{m} \bar{Y}}{\partial \theta^{m}}+\bar{f}(s, \theta) ; \quad \bar{Y}=\left\{N_{x}, N_{z}, \widehat{S}, M_{s}, u_{x}, u_{z}, v, \theta_{s}\right\}^{T} \text {, }
$$

где $N_{x}, N_{z}$ - радиальное и осевое усилия; $u_{x}, u_{z}$ - аналогичные перемещения; $\widehat{S}$ - сдвигающее усилие; $M_{s}$ - меридиональный изгибающий момент; $v$ - окружное перемещение; $\theta_{s}$ - угол поворота нормали; $s$ - длина дуги меридиана; $\theta$ - центральный угол в сечении радиуса $r(s)$, перпендикулярном оси вращения. Элементы матрицы $A_{m}$ зависят от геометрических и механических характеристик оболочки.

Не для всех моделей оболочек это разделение возможно, но для классической теории переменные разделяются. Таким образом, представляя переменные и нагрузки с использованием рядов Фурье [10,19], приходим к системе обыкновенных дифференциальных уравнений

$$
\frac{d \bar{Y}}{d s}=A(s) \bar{Y}+\bar{f}(s) ; \quad\left(s_{0} \leq s \leq s_{L}\right)
$$

с граничными условиями

$$
B_{1} \bar{Y}\left(s_{0}\right)=\bar{b}_{1} ; \quad B_{2} \bar{Y}\left(s_{L}\right)=\bar{b}_{2} .
$$

Здесь $\bar{Y}=\left\{Y_{1} Y_{2} \ldots . . Y_{\mathrm{n}}\right\}$ - вектор функция искомого решения; $A(s), B_{1}, B_{2}$ - заданные матрицы порядка соответственно $n \times n, k \times n, k \times n(k=n / 2) ; \bar{f}(s), \bar{b}_{1}, \bar{b}_{2}$ - заданные векторы.

Данную систему можно проинтегрировать с использованием ортогонализации по С. К. Годунову [21].

\section{3. Контактные задачи теории оболочек}

Одной из разновидностей задач для оболочек, которые часто встречаются при расчете различных технических объектов, являются задачи с подвижными границами контактные задачи. Прикладное значение контактных задач трудно переоценить, поскольку любая конструкция обычно представляют собой, совокупность элементов, каким-либо образом закрепленных в пространстве и контактирующих при эксплуатации. В предисловии монографии Кеннета Джонсона [33] отмечено, что экономические потери от разрушения контактирующих элементов в США составляют более 50 млрд долларов в год. Достаточно часто области контакта и распределение контактного давления между элементами наперед не известны. При этом экспериментальными методами распределение контактной нагрузки точно определить достаточно трудно. Поэтому приходится ставить и решать самые разнообразные контактные задачи, которые будут определять напряжённое состояние контактирующих элементов.

Впервые задача о контакте упругих тел, первоначально соприкасавшихся в точке, была поставлена и решена Генрихом Герцем. Можно сказать, что с появлением его классической работы в контакте упругих тел [34] начала формироваться область механики деформируемого твердого тела - механика контактного взаимодействия. 
Среди разнообразных методов, применяемых при решении задач контактного взаимодействия оболочек, можно выделить основные: это - метод сопряжения решений дифференциальных уравнений, метод интегральных уравнений, методы редуцированных систем и вариационных неравенств и асимптотические методы [35, 36, 37, 38, 39, 40].

При решении аналитическими методами чаще всего используется метод приведения задачи к интегральным уравнениям Фредгольма первого рода. Если интегральное уравнение получено в рамках гипотез Кирхгофа-Лява, то оно имеет лишь обобщенное решение - контактная реакция представляет собой сосредоточенные на границе области контакта силы.

При использовании теорий оболочек, учитывающих поперечный сдвиг, решение можно получить только в классе функций, имеющих разрывы первого рода. При заданной области контакта терпит разрыв поперечная сила. При неизвестной области (на границе этой области), контактное давление конечно. Однако известно, что при решении задач с использованием уравнений теорий упругости на границе зоны сосредоточенные реакции не возникают.

Контактную задачу теории оболочек можно сделать математически корректной методами регуляризации, сущность которых заключается в переходе от уравнений Фредгольма первого рода к уравнениям Фредгольма второго рода. В контактных задачах получили распространение методы физической регуляризации, которые основаны на уточнении физической постановки задачи. Один из них заключается в том, что в зоне контакта вводится упругий слой, учитывающий реальные свойства микрогеометрии контактирующих поверхностей. В этом подходе параметр регуляризации определяется экспериментально и характеризует свойства поверхностей.

Другой метод физической регуляризации основан на учете в зоне контакта обжатия оболочки по толщине, которым классическая теория пренебрегает. Здесь коэффициент регуляризации имеет другой физический смысл. Учет изменения расстояния между срединной и внешней поверхностями оболочки под действием контактного давления дает необходимый вклад в интегральное уравнение и приводит его к уравнению Фредгольма второго рода.

Одним из эффективных способов решения контактных задач для оболочек вращения является метод виртуальных элементов [41, 42]. На рис. 4 представлена расчетная схема для оболочки, лежащей на опоре ложементного типа.

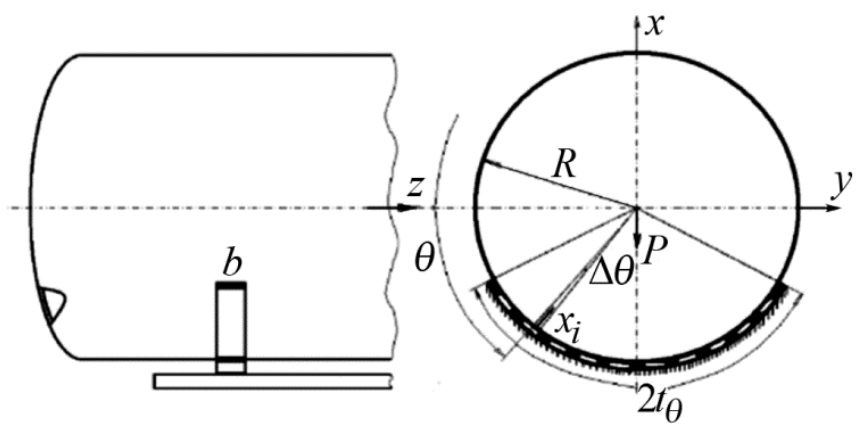

Рис. 4. Расчетная схема для задачи передачи нагрузки от котла вагона-цистерны на лежневые опоры

Данная схема моделирует работу конструкции вагона-цистерны, изображенной на рис. 2. Задача заключается в нахождении контактной нагрузки, области контакта и определения напряженного состояния оболочки цистерны от внешних нагрузок и найденной контактной нагрузки, действующих на конструкцию.

Суть метода в том, что неизвестная область контакта покрывается виртуальными элементами (рис. 5). 


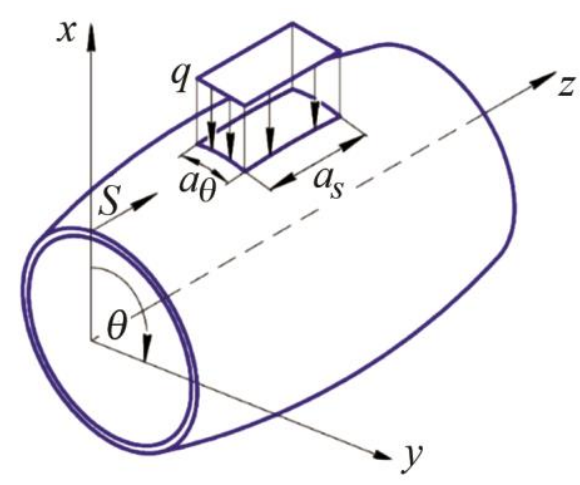

Рис. 5. Оболочка нагружена локальной нагрузкой, распределенной на виртуальном элементе

На элементах принимается постоянное контактное давление. В отличие от конечного элемента количество виртуальных элементов не увеличивает размер матрицы жесткости. Также размеры элемента по направляющей и образующей являются независимыми, в отличие от размеров, принимаемых в методе конечных элементов. Это большое преимущество, которое позволяет сохранять вычислительные ресурсы для многих задач.

Затем строятся канонические уравнения сопряжения оболочки с основанием (опорой). Для одномерного случая (длина контакта по образующей задана) такая система имеет вид [19, 23]:

$$
\begin{gathered}
\sum_{i=1}^{N} \delta_{1 i} X_{i}-Z \cos \theta_{1}+\Delta_{P 1}=0 \\
\sum_{i=1}^{N} \delta_{2 i} X_{i}-Z \cos \theta_{2}+\Delta_{P 2}=0 \\
\ldots \ldots \ldots \ldots \ldots \ldots \ldots \ldots \ldots \ldots \ldots \ldots \ldots \ldots \ldots \ldots \\
\sum_{i=1}^{N} \delta_{N i} X_{i}-Z \cos \theta_{N}+\Delta_{P N}=0 \\
\sum_{i=1}^{N} \cos \theta_{i} X_{i}=P, \quad N=\frac{\pi R_{1}}{a_{\theta}} .
\end{gathered}
$$

Здесь $\delta_{i j}-$ перемещение в основной системе координат по направлению $i$ связи от единичного усилия, введенного по направлению отброшенной $j$ связи; $Z \cos \theta_{i}-$ перемещение в основной системе по направлению отброшенной $i$ связи, происходящее от единичного перемещения по направлению введенной связи; $\Delta_{P i}$ - перемещение по направлению $i$ связи, вызванное действием заданной внешней нагрузки на оболочку; $P$ - внешняя нагрузка, действующая определенным образом на оболочку; $X_{i}-$ неизвестные усилия взаимодействия.

В общем случае из-за разности радиусов между оболочкой и основанием может существовать первоначальный зазор $\Delta_{R}$. Также в некоторых случаях между оболочкой и основанием может присутствовать упругий слой. Следовательно, учитывая эти факторы, уравнения примут вид:

$$
\sum_{i=1}^{\mathrm{N}} \delta_{1 i} X_{i}+D X_{1}-Z \cos \theta_{1}+\Delta_{P 1}+\Delta_{R 1}=0
$$




$$
\begin{gathered}
\sum_{i=1}^{\mathrm{N}} \delta_{N i} X_{i}+D X_{\mathrm{N}}-Z \cos \theta_{\mathrm{N}}+\Delta_{P \mathrm{~N}}+\Delta_{R \mathrm{~N}}=0 \\
\sum_{i=1}^{\mathrm{N}} \cos \theta_{i} X_{i}-P=0 .
\end{gathered}
$$

Здесь $\Delta_{R i}$ - величина зазора по направлению $i$ связи; $D$ - оператор, связывающий реактивное усилие $i$ точки поверхности основания (прокладки) и ее перемещение. Оператор $D$ является аналогом параметра регуляризации, применяемым в аналитических методах решения контактных задач теории оболочек. При этом предположим, что оператор $D$ состоит из двух слагаемых:

$$
D=D_{1}+D_{2},
$$

где оператор $D_{1}$ определяет изменение толщины оболочки за счет обжатия, а оператор $D_{2}$ характеризует упругие свойства прокладок.

Решая данную систему уравнений и применяя итерационные процедуры, находим распределение контактного давления и область контакта. С использованием дискретных рядов Фурье найденная нагрузка аппроксимируется как

$$
q_{f}=\frac{a_{0}}{2}+\sum_{k=1}^{\infty}\left(a_{K} \cos k \theta+b_{K} \sin k \theta\right)
$$

Коэффициенты разложения представим следующим образом:

$$
\begin{aligned}
& a_{K}=\frac{2}{N}\left[\frac{1}{2} q_{\Sigma i}+\sum_{i=1}^{N-1} q_{\Sigma i} \cos \frac{\pi k}{N} i+\frac{1}{2}(-1)^{K} q_{\Sigma N}\right], \\
& b_{k}=0 .
\end{aligned}
$$

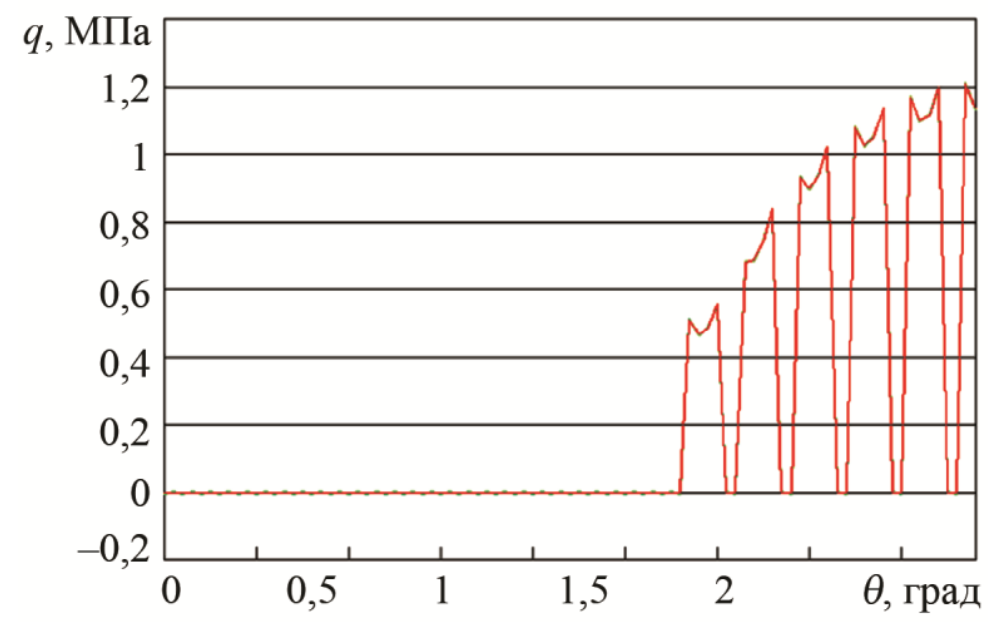

Рис. 6. Аппроксимация контактного давления на половине области контакта для случая взаимодействия оболочки с несплошным упругим основанием

На рис. 6 показана ее аппроксимация на половине области контакта. При этом определяется и контролируется невязка аппроксимации. Такое представление контактной нагрузки необходимо для определения напряженного состояния оболочки от найденной контактной нагрузки. 


\section{4. Результаты по исследованию тонкостенных конструкций}

1. В уравнениях, описывающих контакт оболочки с основанием, использовалось понятие виртуальный элемент, на котором приложена некоторая постоянная сила. Оболочка под действием этой локальной силы деформируется. Была проведена оценка деформации с использованием предлагаемой методики и известных коммерческих вычислительных комплексов ANSYS, COSMOS. Получено хорошее совпадение результатов расчета (по напряжениям и перемещениям) по предлагаемой численно-аналитической методике и методом конечных элементов.

2. Проведено обобщение метода для задачи при возникающей боковой нагрузке (рис. 7).

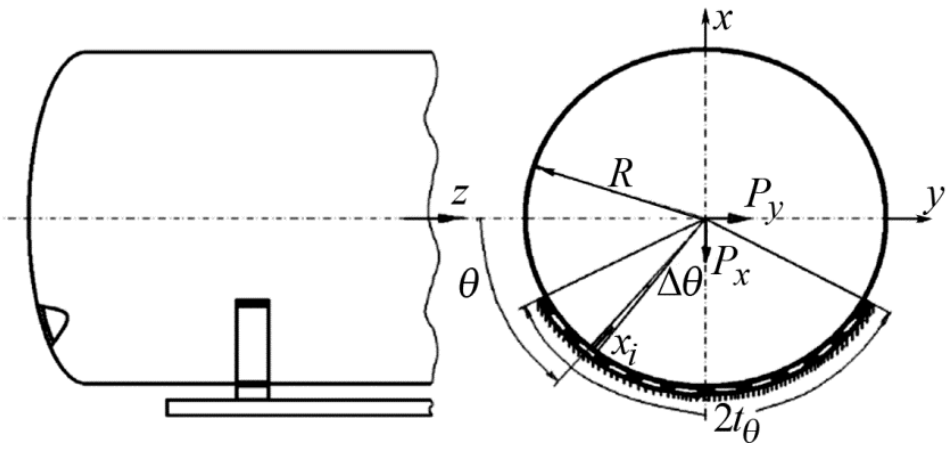

Рис. 7. Контактная задача для оболочки при нагрузке, действующей в вертикальной и горизонтальной плоскости

Определено контактное давление для тонкой изотропной оболочки, лежащей на не сплошном упругом или жестком основании. При этом оболочка нагружена системой массовых сил, включая поперечную нагрузку, которые вызывают несимметричное контактное давление. Данная задача моделирует передачу нагрузки от котла вагона-цистерны на лежневые опоры при прохождении вагоном поворота, т. е. с учетом центробежной силы. Вычисленная, несимметричная относительно вертикальной оси контактная нагрузка, представлена на рис. 8.

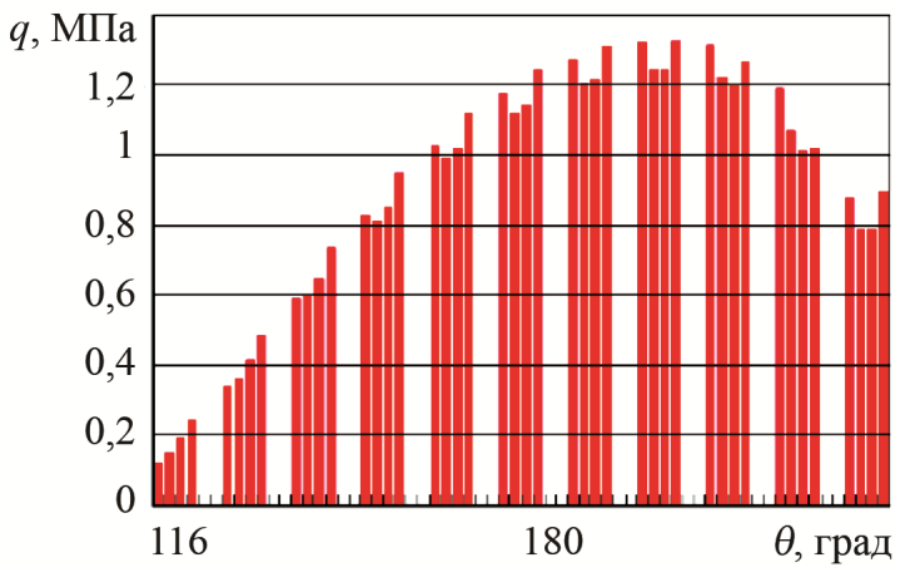

Рис. 8. Распределение контактного давления для случая взаимодействия оболочки с упругим основанием с учетом поперечной нагрузки

3. Поскольку получаемая контактная нагрузка не симметричная, то предложено ее новое разложение в дискретный ряд Фурье по синусам и косинусам. На рис. 9 показана ее аппроксимация на половине области контакта. Невязка аппроксимации составляет менее $0,1 \%$. 


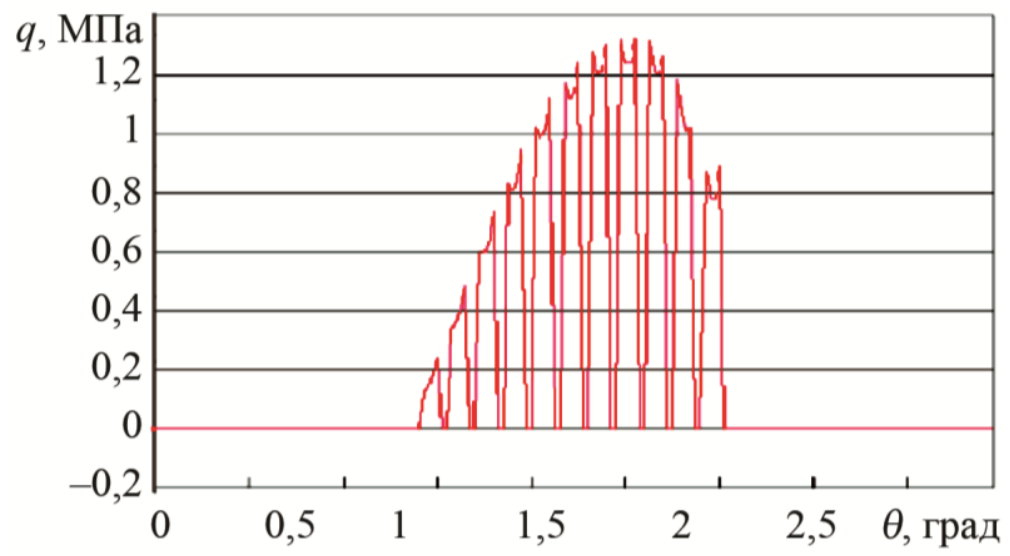

Рис. 9. Аппроксимация контактного давления для случая взаимодействия оболочки с упругим основанием при вертикальной и поперечной нагрузке

4. Проведено обобщение метода для задачи взаимодействия оболочки по неизвестной области контакта в двух координатных направлениях по направляющей и образующей. При этом область основания может быть несплошной, как на рис. 4. На рис. 10-11 показано распределение контактного давления по двумерной сплошной области, на рис. 12-13 распределение контактного давления по двумерной области в области имеющей разрывы.

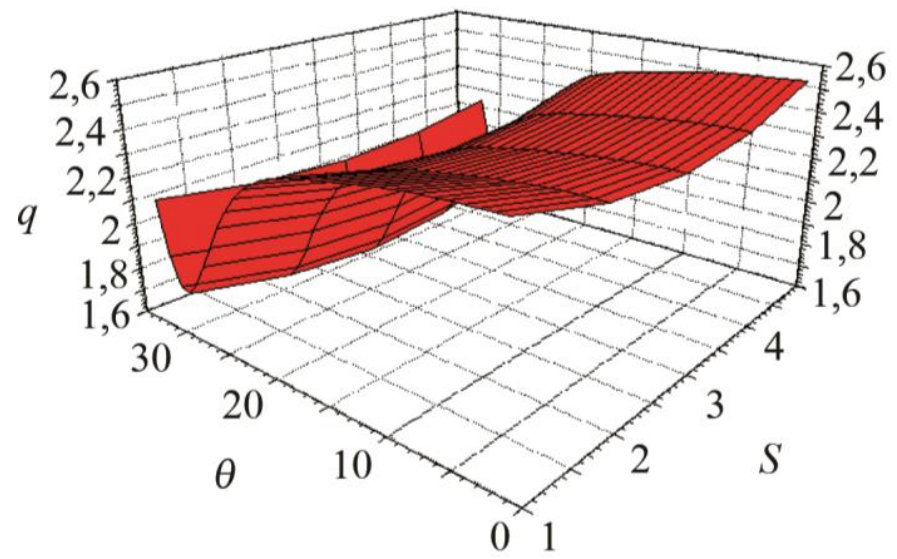

Рис. 10. Распределение контактных напряжений на половине области контакта в случае взаимодействия со сплошным упругим основанием

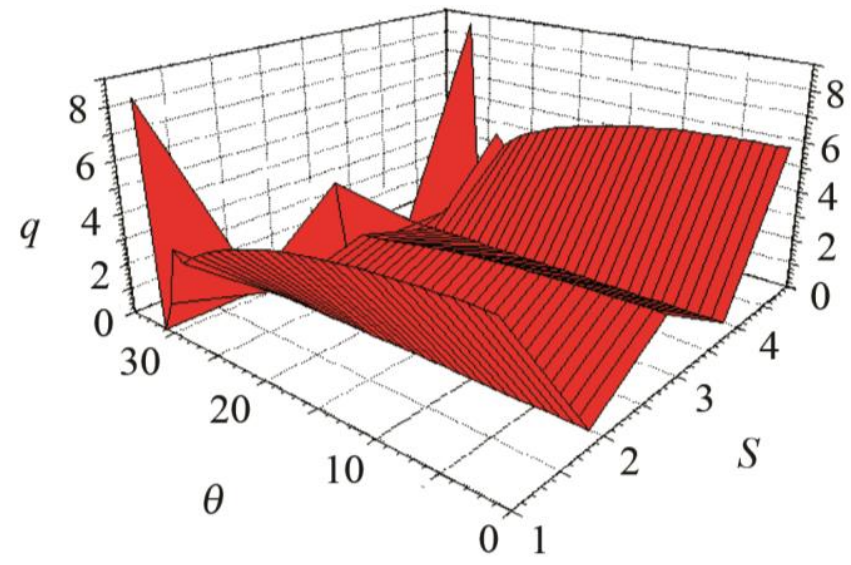

Рис. 11. Распределение контактных напряжений на половине области контакта в случае взаимодействия со сплошным жестким основанием 


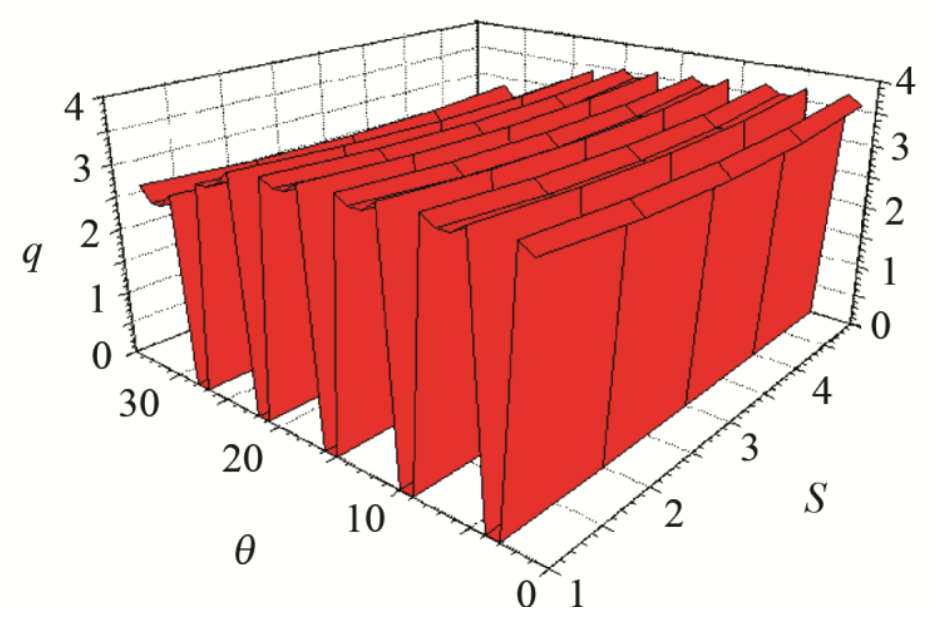

Рис. 12. Распределение контактных напряжений на половине области контакта в случае взаимодействия с несплошным упругим основанием

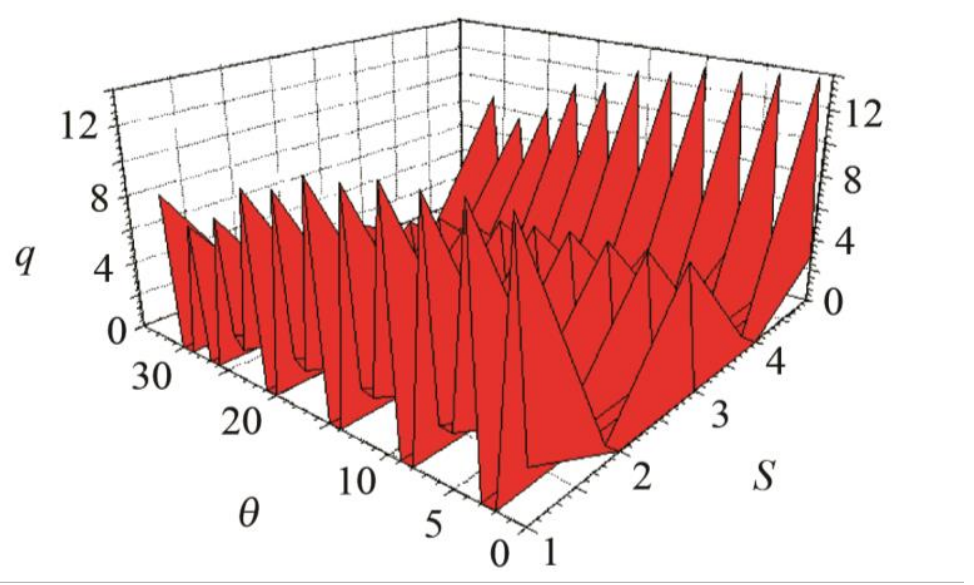

Рис. 13. Распределение контактных напряжений на половине области контакта в случае взаимодействия с несплошным жестким основанием

5. Метод виртуальных элементов, обобщен для решения контактных задач для цилиндрических оболочек, лежащих на основании с поверхностью несогласованной формы. Контакт оболочки может происходить как по согласованной, так и по несогласованной поверхности контакта. Контакт называется согласованным, если поверхности обоих тел в недеформированном состоянии точно «подогнаны» друг к другу. Тела, имеющие различные по форме профили, называются несогласованными. Тела несогласованной формы первоначально вступают в контакт в точке или по линии. Под действием внешней нагрузки они деформируются в окрестности точки начального контакта и приходят в соприкосновение по некоторой конечной области.

6. Показано, что существует аналогия между линейными интегральными уравнениями, описывающими одномерную контактную задачу для оболочки и разрешающими линейными алгебраическими уравнениями метода виртуальных элементов. Доказано, что уравнение Фредгольма второго рода, определяющее распределение контактного давления в одномерной постановке, совпадает с точностью до констант с уравнениями по предлагаемой методике. Численно построенная функция податливости $\delta_{i j}$ является аналогом функции Грина $G$. Необходимо добавить, что только для ограниченного класса оболочек (прямоугольных пластин, круглых пластин, сферических оболочек, цилиндрических оболочек бесконечной длины) существуют аналитические выражения для функции Грина. Для оболочки вращения 
конечной длины с переменными параметрами вдоль образующей, построить аналитическую функцию Грина невозможно. Но достаточно просто ее вычислить численно, используя выше изложенный численно-аналитический метод.

7. Определено напряженно-деформированное состояние оболочечной конструкции вагона-цистерны (рис. 2), лежащей на опорах ложементного типа, при воздействии нормативных нагрузок разного вида. При расчете использован метод конечных элементов и использован оболочечный конечный элемент типа shell. Расчетная схема конструкции представлена на рис. 14.

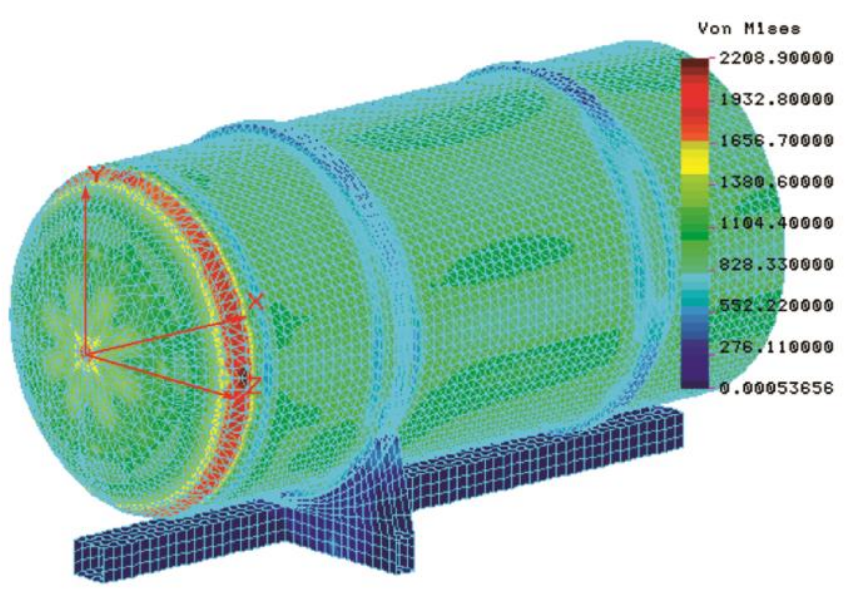

Рис. 14. Напряженное состояние вагона-цистерны при расчете на крупной конечно-элементной сетке

Ресурс нерегулярно нагруженной конструкции вагона-цистерны, работающей в области многоцикловой усталости, оценен по линейной гипотезе накопления усталостных повреждений. Предложен альтернативный подход к определению долговечности конструкции, основанный на дополнительных нестандартных экспериментах с образцами материала конструкции и законом нелинейного суммировании повреждений. Результат по определению долговечности конструкции вагона-цистерны представлен на рис. 15.

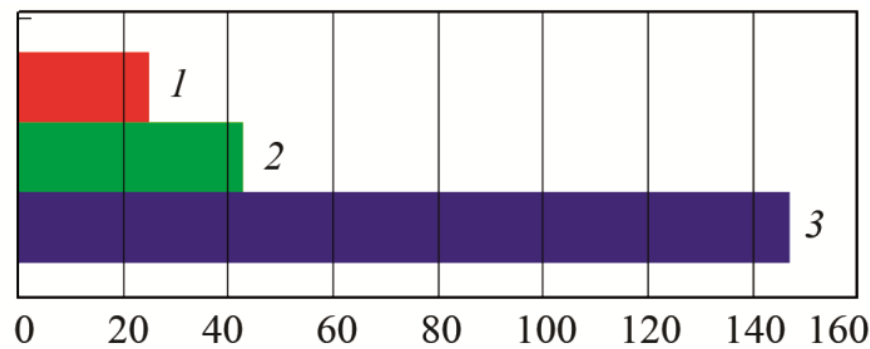

Рис. 15. Оценка долговечности вагона, годы: 1 - гарантийный срок службы;

2 - срок, рассчитанный по методике нелинейного суммирования напряжений; 3 - срок, рассчитанный по стандартной методике

8. Разработана математическая модель, позволяющая оценить сопротивление разрушению кабины локомотива с учетом буферного устройства (рис. 16). 


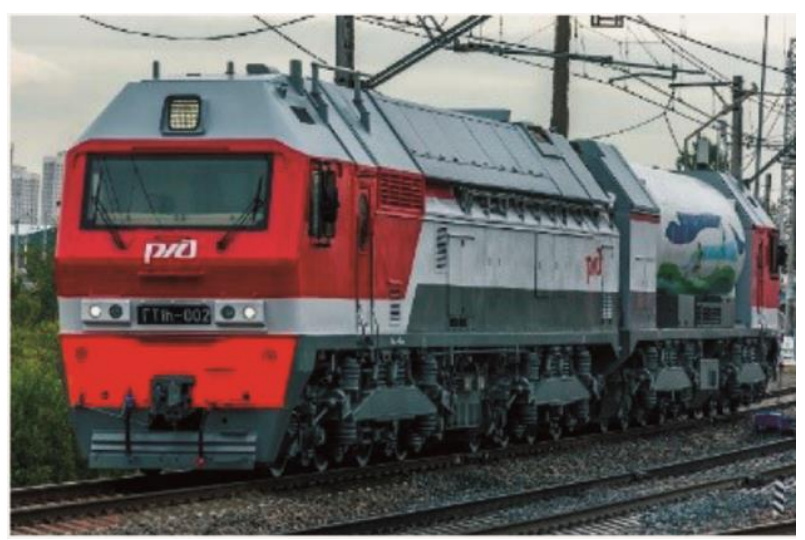

Рис. 16. Кабина локомотива

На примере расчета кабины локомотива при наезде на препятствие предложен подход к определению параметров области безопасной эксплуатации конструкции. Для несущей конструкции балочно-стержневого типа предложен вариант нахождения области безопасной эксплуатации при действии сверхнормативной нагрузки. Рассмотрено нагружение силового тонкостенного каркаса кабины локомотива при наезде на препятствие (рис. 17).

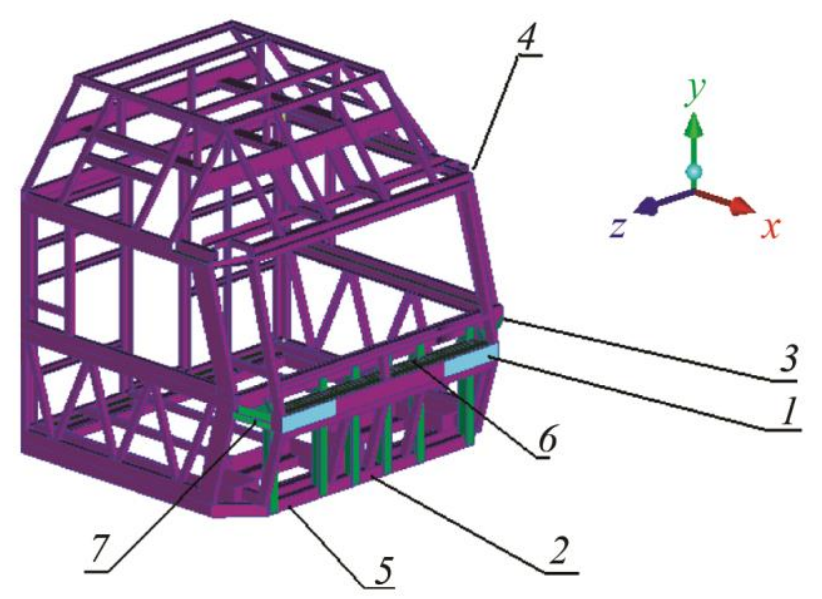

Рис. 17. Расчетная схема силового каркаса кабины локомотива: 1 - лобовая балка; 2 - крепящие элементы; 3 - силовой пояс; 4 - верхняя обвязка кабины; 5 - нижняя обвязка кабины; 6 - набор сотовых элементов; 7 - боковые упоры

При расчете использован метод конечных элементов и использован оболочечный конечный элемент типа shell. Предложенная детерминированная инженерная оценка напряженного состояния сложной конструкции позволяет определить в трехмерном пространстве неизвестных параметров препятствия предельную поверхность, которая должна ограничивать область безопасной эксплуатации локомотива (рис. 18). На примере расчета кабины локомотива показано, каким образом необходимо обосновать требование сохранения ее прочности и тем самым сохранить безопасность работы машинистов и дорогостоящего оборудования кабины. 


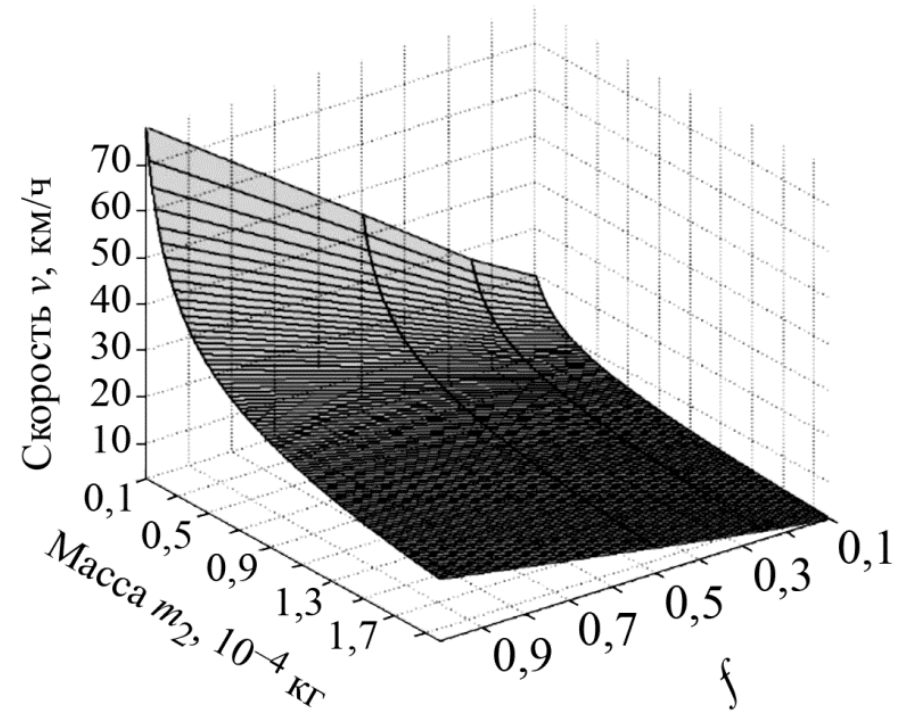

Рис. 18. Область безопасной эксплуатации кабины локомотива

\section{5. Заключение}

Полученные в последнее время вышеперечисленные результаты фундаментальных и прикладных исследований для тонкостенных конструкций с использованием методов и подходов механики оболочек опубликованы в следующих работах [24, 41-63].

\section{Литература}

1. Ляв А. Математическая теория упругости. - М.; Л. : ОНТИ, 1935. - 674 с.

2. Власов В. 3. Общая теория оболочек и ее приложения в технике. - М.; Л. : Гостехиздат, 1949. - $784 \mathrm{c.}$

3. Гольденвейзер А. Л. Теория упругих тонких оболочек. - 2-е изд. испр. и доп. М. : Наука, 1976. - 512 с.

4. Лурье А. И. Статика тонкостенных упругих оболочек. - М.; Л. : Гостехиздат, 1947. $252 \mathrm{c}$.

5. Новожилов В. В. Теория тонких оболочек. - Л. : Судостроение, 1962. - 431 с.

6. Тимошенко С. П., Войновский-Кригер С. Пластинки и оболочки / пер. с англ. М. : Физматгиз, 1963. -635 с.

7. Флюгге В. Статика и динамика оболочек / пер. со второго нем. изд. инж. В. Л. Шадурского. - М. : Госстройиздат, 1961. - 306 с.

8. Черных К. Ф. Линейная теория оболочек. - Л. : Изд-во ЛГУ, 1962. - Ч. 1. - 274 с.

9. Черных К. Ф. Линейная теория оболочек. - Л. : Изд-во ЛГУ, 1964. - Ч. 2. - 396 с.

10. Григоренко Я. М., Василенко А. Т. Методы расчета оболочек : в 5 т. - Киев : Наукова думка, 1981. - Т. 4 : Теория оболочек переменной жесткости. - 544 с.

11. Вольмир А. С., Куранов Б. А., Турбаивский А. Т. Статика и динамика сложных структур. - М. : Машиностроение, 1989. - 248 с.

12. Григоренко Я. М. Изотропные и анизотропные слоистые оболочки вращения переменной жесткости. - Киев : Наукова думка, 1973. - 228 с.

13. Рогалевич В. В. Коллокационные методы. Сущность. Примеры. - Екатеринбург : Издательство АМБ, 2001. - 298 с.

14. Крауч С., Старфилд А. Методы граничных элементов в механике твердого тела / пер. с англ. - М. : Мир, 1987. - 328 с.

15. Бреббия К., Уокер С. Применение метода граничных элементов в технике / пер. с англ. - М. : Мир, 1982. - 248 с. 
16. Артюхин Ю. П., Грибов А. П. Решение задач нелинейного деформирования пластин и пологих оболочек методом граничных элементов. - Казань : Фэн, 2002. - 199 с.

17. Бенерджи П., Баттерфилд Р. Методы граничных элементов в прикладных науках / пер. с англ. - М. : Мир, 1984. - 496 с.

18. Канторович Л. В., Крылов В. И. Приближенные методы высшего анализа. М. : Физматгиз, 1948. - 708 с.

19. Механика композитов : в 12 т. / Я. М. Григоренко, А. Т. Василенко, И. Г. Емельянов, Н. Н. Крюков, Ю. Н. Немиш, Н. Д. Панкратова, Б. Л. Пелех, Г. Г. Влайков, А. В. Максимук, Г. П. Урусова / ред. А. Н. Гузь. - К. : Наук. думка, 1999. - Т. 8 : Статика элементов конструкций / НАН Украины. Ин-т механики. - Киев : А.С.К., 1999. - 379 с.

20. Статика и динамика тонкостенных оболочечных конструкций / А. В. Кармишин, В. А. Лясковец, В. И. Мяченков, А. Н. Фролов. - М. : Машиностроение, 1975. - 76 с.

21. Годунов С. К. О численном решении краевых задач для систем линейных обыкновенных дифференциальных уравнений // Успехи математических наук. - 1961. - Т. 16. - № 3. C. 171-174.

22. Григоренко Я. М., Влайков Г. Г., Григоренко А. Я. Численно-аналитическое решение задач механики оболочек на основе различных моделей. - Киев : Академпериодика, 2006. - 472 с.

23. Емельянов И. Г. Контактные задачи теории оболочек. - Екатеринбург : УрО РАН, 2009. - 185 c.

24. Емельянов И. Г., Миронов В. И. Долговечность оболочечных конструкций. - Екатеринбург : УpO РAH, 2012. $-224 \mathrm{c}$.

25. Галлагер Р. Метод конечных элементов. Основы / пер. с англ. - М. : Мир, 1984. $428 \mathrm{c}$.

26. Голованов А. И., Тюленева О. Н., Шигабутдинов А. Ф. Метод конечных элементов в статике и динамике тонкостенных конструкций. - М. : ФИЗМАТЛИТ, 2006. - 392с.

27. Рикардс Р. Б. Метод конечных элементов в теории оболочек и пластин. - Рига : Зинатне, 1988. - 284 c.

28. Зенкевич О. К. Метод конечных элементов в технике. - М. : Мир, 1975. - 541с.

29. Новожилов И. И. Вопросы механики сплошной среды. - Л. : Судостроение, 1989. $400 \mathrm{c}$.

30. Емельянов И. Г., Ефимов В. П., Кузнецов А. В. Модель напряженно-деформированного состояния котла вагона-цистерны с усовершенствованной схемой опирания на раму // Тяжелое машиностроение. - 2005. - № 8. - С. 44-49.

31. Емельянов И. Г., Миронов В. И., Кузнецов А. В. Определение напряженного состояния и ресурса оболочечной конструкции // Проблемы машиностроения и надежности машин. - 2007. - № 5. - С. 57-65.

32. Василенко А. Т., Голуб Г. П., Григоренко Я. М. Расчет параметров напряженного состояния конструктивных элементов из композиционных материалов на основе оболочечных моделей // Расчеты на прочность : сборник научных статей. - Вып. 30. - М. : Машиностроение, 1989. C. 87-96.

33. Джонсон К. Механика контактного взаимодействия / пер. с англ. - М. : Мир, 1989. $510 \mathrm{c}$.

34. Hertz H. Über die Berührung fester elastischer Körper // Journal für die reine und angewandte Mathematik. - 1881. - num. 92. - P. 156-171.

35. Григолюк Э. И., Толкачев В. М. Контактные задачи теории пластин и оболочек. М. : Машиностроение, 1980. - 411 с.

36. Кантор Б. Я. Контактные задачи нелинейной теории оболочек вращения. - Киев : Наукова думка, 1990. - 136 с. 
37. Артюхин Ю. П., Малкин С. А. Аналитические и численные методы решения интегральных уравнений в задачах упругого воздействия тел. - Казань : Казанский гос. ун-т, 2007. - 292 c.

38. Моссаковский В. И., Гудрамович В. С., Макеев Е. М. Контактное взаимодействие элементов оболочечных конструкций. - Киев : Наукова думка, 1988. - 288 с.

39. Образцов И. Ф., Нерубайло Б. В., Ольшанский В. П. Оболочки при локализованных воздействиях (обзор работ, основные результаты и направления исследований). - М., 1988. 192 с. - Деп. в ВИНИТИ 12.02.88.

40. Пелех Б. Л., Максимук А. В., Коровайчук И. М. Контактные задачи для слоистых элементов конструкций и тел с покрытиями. - Киев : Наукова думка, 1988. - 280 с.

41. Емельянов И. Г., Кузнецов А. В. Применение виртуальных элементов при определении напряженного состояния оболочек вращения // Вычислительная механика сплошных сред. - 2014. - Т. 7, по. 3. - С. 245-252.

42. Emel'yanov I. G. Investigation into the Contact Interaction between Shell and Base with Notches // Journal of Machinery Manufacture and Reliability. - 2015. - Vol. 44, no. 3. P. 263-270. - DOI: 10.3103/S1052618815030048.

43. Emel'yanov I. G., Mironov V. I., Kuznetsov A. V. Evaluation of the life of a shell construction lying on supports // Journal of Machinery Manufacture and Reliability . - 2010. - Vol. 39, iss. 1, pp. 83-88. - DOI: 10.3103/S1052618810010139.

44. Миронов В. И., Емельянов И. Г. Метод полных диаграмм в расчетах на сопротивление усталости // Международная научно-техническая конференция «Прочность материалов и элементов конструкций», Киев, 28-30 сентября 2010 г. : труды конференции / отв. ред. В. Т. Трощенко. - Киев : ИПП НАНУ, 2011. - С. 697-704.

45. Емельянов И. Г., Миронов В. И., Кузнецов А. В. Циклическая долговечность оболочки вагона - цистерны // Международная научно-техническая конференция «Прочность материалов и элементов конструкций», Киев, 28-30 сентября 2010 г. : труды конференции / отв. ред. В. Т. Трощенко. - Киев : ИПП НАНУ, 2011. - С. 836-843.

46. Контактное взаимодействие котла вагона-цистерны с лежневыми опорами / И. Г. Емельянов, В. И. Миронов, А. В. Кузнецов, А. В. Якушев // Известия Самарского научного центра Российской академии наук. - 2011. - Том 13, № 1-2. - С. 436-439.

47. Емельянов И. Г. Миронов В. И., Якушев А. В. Контактная задача в расчетах усталостной прочности элементов вагона-цистерны // Транспорт Урала. - 2011. - № 3 (30). - С. 49-55. 48. Разработка экспресс-метода для контроля свойств вагонных сталей / И. Г. Емельянов, В. И. Миронов, А. В. Якушев, О. А. Лукашук // Транспорт Урала. - 2012. - № 2 (33). C. $13-17$.

49. Barashkova E., Emelyanov I. Stress State of Shells under Arbitrary Load // Proceedings of the 5th WSEAS International Conference on Finite Differences - Finite Elements - Finite Volumes - Boundary Elements (F-and-B'12), Prague, Czech Republic, September 24-26, 2012. Prague : WSEAS Press, 2012.- P. 33-37.

50. Emelyanov I. G., Mironov V. I. Contact problem for a shell considering the transverse load // Journal of Machinery Manufacture and Reliability. - 2013. - Vol. 42, no. 1. - P. 36-40. DOI: $10.3103 / \mathrm{S} 1052618813010056$.

51. Barashkova E. A., Emelyanov I. G. Determination of the Stress State of Shells under Local Load // Mathematical methods and optimization techniques in engineering : proceedings of the First International Conference on Optimization Techniques in Engineering (OTENG '13), Antalya, Turkey, October 8-10, 2013. - 2013. - P. 109-113.

52. Емельянов И. Г., Кузнецов А. В., Миронов В. И. Математическая модель, описывающая напряженное состояние кабины газотурбовоза при встрече с препятствием // Транспорт Урала. - 2013. - № 4 (39). - С. 71-74. 
53. Emelyanov I. G., Kuznetsov A. V. The stressed state of shell structures under local loads // Journal of Machinery Manufacture and Reliability. - 2014. - Vol. 43, no. 1. - P. 42-47. DOI: $10.3103 / \mathrm{S} 1052618814010051$.

54. Емельянов И. Г., Кузнецов А. В., Миронов В. И. Определение долговечности элементов конструкций вагона-цистерны // Надежность и долговечность машин и сооружений. 2014. - Вып. 38. - С. 45-54.

55. Mironov V. I., Kuznetsov A. V., Emel'yanov I. G. Consideration of cyclic degradation of the material and abnormality of the surface layer mechanical properties in calculating the life of a plate with an opening // Strength of Materials. - 2014. - Vol. 46, no. 5. - P. 638-643. DOI: $10.1007 / \mathrm{s} 11223-014-9594-y$.

56. Yemel'yanov I. G. Application of discrete Fourier series to the stress analysis of shell structures // Computational Continuum Mechanics. - 2015. - Vol. 8, no. 3. - P. 245-253. DOI: $10.7242 / 1999-6691 / 2015.8 .3 .20$.

57. Emel'yanov I. G. Mironov V. I. Kuznetsov A. V. Estimation of the Fracture Strength of a Spatial Beam-Rod Structure Notches // Journal of Machinery Manufacture and Reliability. 2015. - Vol. 44, no. 5. - P. 449-454. - DOI: 10.3103/S1052618815050076.

58. Emel'yanov I. G. Mironov V. I. Kuznetsov A. V. On An Approach to the Evaluation of the Strength of a Spatial Rod System under Impact Loading // Diagnostics, Resource and Mechanics of materials and structures. - 2015. - Iss. 2. - P. 16-23. - URL: http://dream-journal.org/issues/ 2015-2/2015-2_24.html (accessed 05.05.2015).

59. Emel'yanov I. G., Mironov V. I., Kuznetsov A. V. The definition of the resistance area boundaries for a locomotive operator cab under non-regulated loads // AIP Conf. Proc. - 2016. Vol. 1785. - P. 040014. - DOI: 10.1063/1.4967071.

60. Emel'yanov I. G. Mironov V. I. Kuznetsov A. V. Determination of safe operating conditions for supporting structures under abnormal loads // Journal of Machinery Manufacture and Reliability. - 2017. - Vol. 46. - No. 5. - P. 136-142. - DOI: 10.3103/S1052618817020042.

61. Emel'yanov I. G., Mironov V. I., Kuznetsov A. V. Vehicle Survivability Calculation // Russian Journal of Construction Science and Technology. - 2017. - Vol. 3, no. 1. - P. 40-44. DOI: $10.15826 /$ rjcst.2017.1.005.

62. Емельянов И. Г., Кузнецов А. В. Определение напряженного состояния тонкостенных конструкций с использованием методов теории оболочек // Транспортные системы и технологии. - 2017. - № 3. - С. 64-78.

63. Emelyanov I. G., Mironov V. I., Kuznetsov A. V. Evaluating the effect of damping structures in the design of a locomotive cab during a collision. - AIP Conference Proceedings. - 2017. Vol. 1915. - P. 040011. - DOI: 10.1063/1.5017359. - URL: http://aip.scitation.org/ toc/apc/1915/1?expanded=1915. 